\title{
Magnetoresistance in a high-mobility two-dimensional electron gas
}

\author{
L. Bockhorn, ${ }^{1}$ P. Barthold, ${ }^{1}$ D. Schuh, ${ }^{2}$ W. Wegscheider, ${ }^{2, *}$ and R. J. Haug ${ }^{1}$ \\ ${ }^{1}$ Institut für Festkörperphysik, Leibniz Universität Hannover, Appelstr. 2, D-30167 Hannover, Germany \\ ${ }^{2}$ Institut für Experimentelle und Angewandte Physik, Universität Regensburg, Universitätsstrasse 31, D-93053 Regensburg, Germany
}

(Received 30 November 2010; published 2 March 2011)

\begin{abstract}
In a high-mobility two-dimensional electron gas (2DEG) in a $\mathrm{GaAs} / \mathrm{Al}_{0.3} \mathrm{Ga}_{0.7}$ As quantum well we observe a strong magnetoresistance. In lowering the electron density, the magnetoresistance gets more pronounced and reaches values of more than $300 \%$. We observe that the huge magnetoresistance vanishes when increasing the temperature. An additional density-dependent factor is introduced to be able to fit the parabolic magnetoresistance to the electron-electron interaction correction.
\end{abstract}

DOI: 10.1103/PhysRevB.83.113301

PACS number(s): 73.43.Qt, 73.23.Ad, 72.10.-d

Since the first observation of the fractional quantum Hall effect (FQHE) $)^{1,2}$ the quality and the mobility of the two-dimensional electron gas (2DEG) increased by more than two orders of magnitude. The increased mobility has allowed not only the observation of the FQHE at many different filling factors and smaller magnetic fields but also many new effects. So, microwave-induced oscillations were observed, which were up to now not fully understood. ${ }^{3-5}$ In weak magnetic fields the increased mobility enabled also the observation of phonon-induced resistance oscillations, which are caused by inelastic scattering between electrons and three-dimensional acoustic phonons. ${ }^{6,7}$ The period of phonon-induced oscillations is tunable by an additional dc electric field. ${ }^{8,9}$ Also a new type of QHE was enabled in high mobility 2DEGs, the re-entrant integer quantum Hall effect (RIQHE). ${ }^{10,11}$ In the regime of the RIQHE the longitudinal resistance between integer filling factors decreases to zero suggesting fractional filling factors, but the corresponding Hall plateaus are quantized at integer values.

Here we will present the observation of a huge magnetoresistance in a high mobility 2DEG which depends strongly on electron density and temperature.

Our samples were cleaved from a wafer of a high-mobility $\mathrm{GaAs} / \mathrm{Al}_{0.3} \mathrm{Ga}_{0.7}$ As quantum well grown by molecular-beam epitaxy. The quantum well has a width of $30 \mathrm{~nm}$ and is Si-doped from both sides. The 2DEG is located $150 \mathrm{~nm}$ beneath the surface and has an electron density of $n_{e} \approx 3.1 \times 10^{11} \mathrm{~cm}^{-2}$ and a mobility of $\mu \approx 11.9 \times 10^{6} \mathrm{~cm}^{2} / \mathrm{Vs}$ in the dark. The specimens are Hall bars with a total length of $1.2 \mu \mathrm{m}$, a width of $w=200 \mu \mathrm{m}$ and a potential probe spacing of $l=275 \mu \mathrm{m}$ [see Fig. 1(a)]. The Hall bars were defined by photolithography and wet etching. Different ungated and gated samples were used for the magnetotransport measurements. In the case of the gated sample there is an additional layer of $600 \mathrm{~nm}$ PMMA between the Hall bar and the metallic top-gate to avoid leakage current. We apply top-gate voltages up to $-6 \mathrm{~V}$ to manipulate the electron density. Our measurements were performed in a dilution refrigerator with a base temperature of $20 \mathrm{mK}$. The measurements were carried out by using low-frequency $(13 \mathrm{~Hz})$ lock-in technique.

Figure 1(a) shows the longitudinal resistance $R_{x x}$ and the Hall resistance $R_{x y}$ vs. magnetic field $B$ to demonstrate the quality of our samples. A series of different fractional quantum Hall states appears for filling factor $v<2$. We observe also the filling factor $v=5 / 2$. Over the range $4<$ $v<6$ the longitudinal resistance decreases to zero between integer filling factors with Hall plateaus being quantized at integer values of $h / 4 e^{2}, h / 5 e^{2}$, and $h / 6 e^{2}$. The here observed phenomenon is the RIQHE.

In Fig. 1(b) the longitudinal resistance $R_{x x}$ vs. magnetic field $B$ is shown for different electron densities $n_{e}$ in the range $-100 \mathrm{mT}$ to $500 \mathrm{mT}$. We observe a peak at zero magnetic field. The strong negative magnetoresistance crosses over to a positive magnetoresistance at about $40 \mathrm{mT}$ for the highest electron concentration. The Shubnikov-de Haas ( $\mathrm{SdH})$ oscillations show for $n_{e}=3.1 \times 10^{11} \mathrm{~cm}^{-2}$ a beating effect, which is also observed till $n_{e}=2.5 \times 10^{11} \mathrm{~cm}^{-2}$. For $n_{e}=$ $2.3 \times 10^{11} \mathrm{~cm}^{-2}$ no beating effect is observable. So, the beating effect disappears by decreasing the electron density. This beating effect for $2.5 \times 10^{11} \mathrm{~cm}^{-2}<n_{e}<3.14 \times 10^{11} \mathrm{~cm}^{-2}$ is attributed to the existence of two $2 \mathrm{D}$ sub-bands. The occupation of the second sub-band occurs above $n_{e}=2.5 \times$ $10^{11} \mathrm{~cm}^{-2}$. This low value is attributed to the double Si-doped quantum well and is lower than previously reported values (see, e.g., Ref. 12). The SdH oscillations start at $110 \mathrm{mT}$. From this onset of the $\mathrm{SdH}$ oscillations we can deduce the density inhomogeneity of our samples ${ }^{13}$ and we find an inhomogeneity of less than $2 \%$.

In Fig. 2 the longitudinal resistivity $\rho_{x x}$ vs. magnetic field $B$ is shown for different top-gate voltages. For each top-gate voltage we achieve a huge magnetoresistance. The longitudinal resistivity decreases almost to a similar background by applying a magnetic field. The difference between this value and the peak is indicated by the percentage (see Fig. 2). The magnetoresistance increases by decreasing the electron density $n_{e}$ and the difference is reaching a value of about $350 \%$. The width of the huge peak in the magnetoresistance increases also with decreasing electron density. The longitudinal magnetoresistance becomes nearly bell-shaped for lower electron densities. At zero magnetic field a small peak is observed on top of the bell-shaped magnetoresistance. This small peak appears for all top-gate voltages.

For gate voltages being larger than $-2 \mathrm{~V}$ the negative magnetoresistance crosses over to a positive magnetoresistance at about $40 \mathrm{mT}$, while for lower top-gate voltages the magnetoresistance does not show such a positive magnetoresistance. Therefore the crossover at $40 \mathrm{mT}$ is attributed to the additional 2D sub-band being occupied for a carrier concentration of 

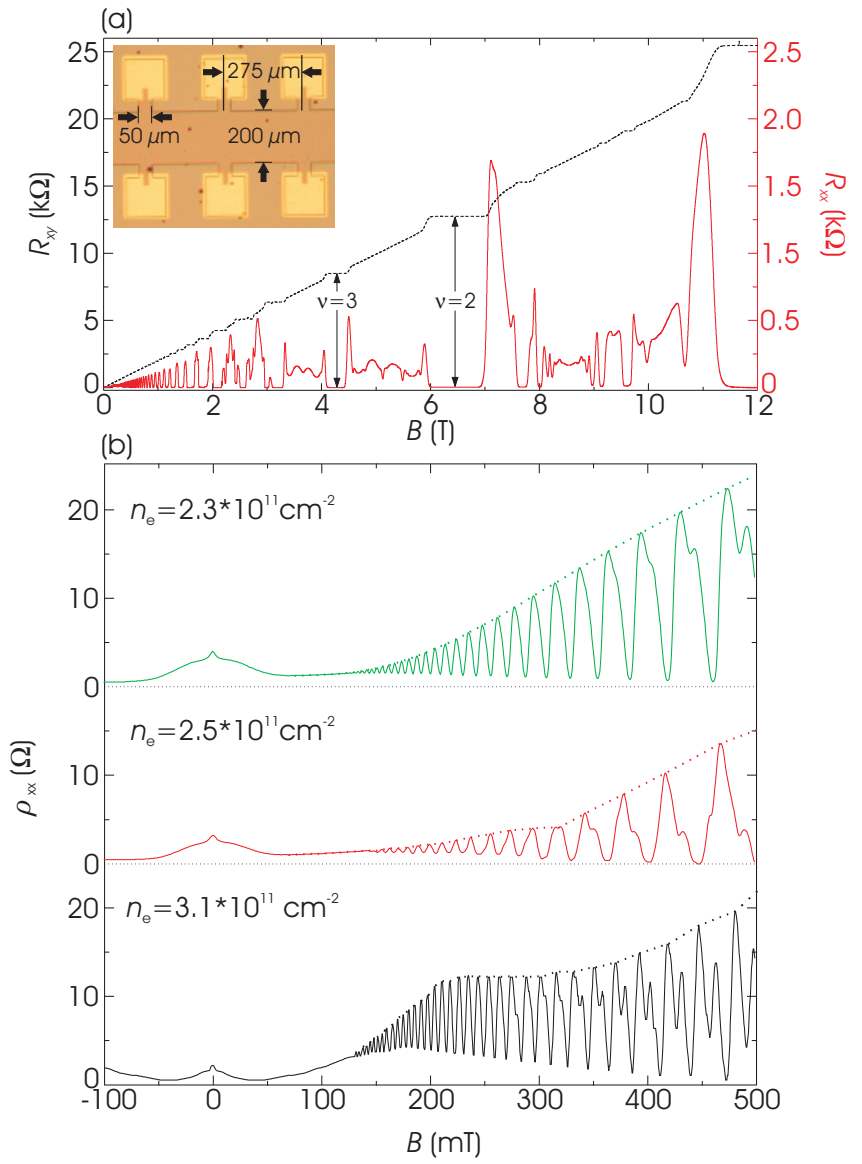

FIG. 1. (Color online) (a) Hall resistance $R_{x y}$ (dashed curve) and longitudinal resistance $R_{x x}$ (solid curve) vs. magnetic field $B$ at $45 \mathrm{mK}$. The electron density is $n_{e} \approx 3.1 \times 10^{11} \mathrm{~cm}^{-2}$ and the mobility is $\mu \approx 11.9 \times 10^{6} \mathrm{~cm}^{2} / \mathrm{Vs}$. The inset shows a part of the Hall bar. (b) The longitudinal resistivity $\rho_{x x}$ vs. magnetic field $B$ for different electron densities in the range $-100 \mathrm{mT}$ and $500 \mathrm{mT}$.

$n_{e}=3.14 \times 10^{11} \mathrm{~cm}^{-2}$. For $n_{e}<2.5 \times 10^{11} \mathrm{~cm}^{-2}$ we do not have any hint to the occupation of the second $2 \mathrm{D}$ sub-band, but we see the huge negative magnetoresistance. Therefore the astonishing behavior of the huge magnetoresistance is not caused by the interaction between different $2 \mathrm{D}$ sub-bands.

The magnetoresistance depends not only strongly on the electron density but also on temperature. In Fig. 3 the longitudinal resistivity $\rho_{x x}$ vs. magnetic field $B$ is shown for a gate voltage of $-4 \mathrm{~V}$ for several temperatures ranging between $100 \mathrm{mK}$ and $800 \mathrm{mK}$. The $\mathrm{SdH}$ oscillations start outside of the shown magnetic field range. Similar to the top-gate voltage dependent measurements we observe the huge magnetoresistance for the lowest temperature, $100 \mathrm{mK}$, and on top of the huge magnetoresistance the small peak at zero magnetic field.

The negative magnetoresistance decreases by increasing the temperature. Meanwhile the small peak at zero magnetic field is left unchanged by increasing the temperature. The temperature independence at $B=0 \mathrm{~T}$ is a sign for the absence of weak localization in our sample. In contrast to the huge magnetoresistance which depends strongly on temperature, the peak at zero magnetic field is temperature independent. Since the mean-free path of our sample is about $113 \mu \mathrm{m}$ and

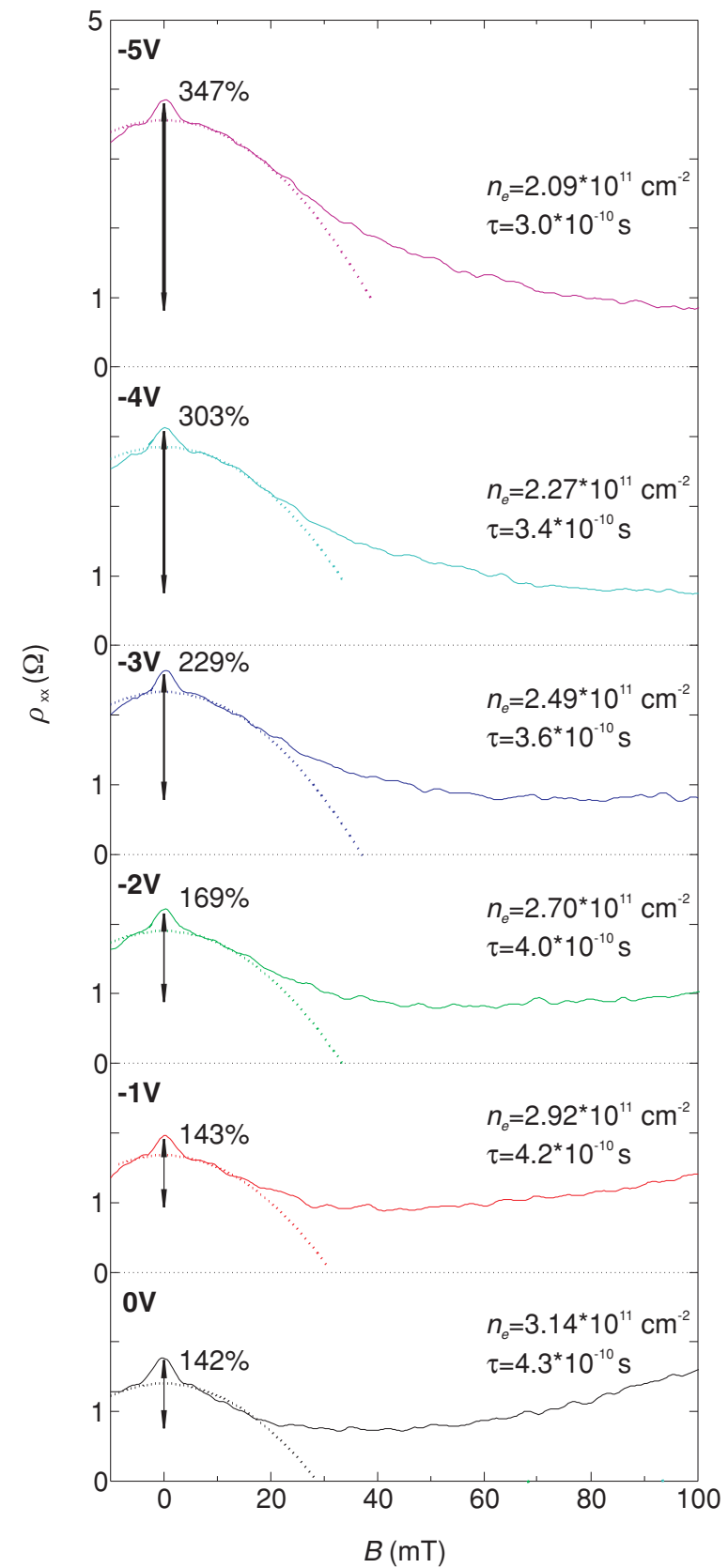

FIG. 2. (Color online) The longitudinal resistivity $\rho_{x x}$ vs. magnetic field $B$ for different top-gate voltages. The different curves have been shifted vertically for clarity.

the Hall bar dimensions are in the range of the mean-free path, one can attribute the observed effect to the influence of ballistic transport. The peak at zero magnetic field is then given by scattering at the edges of the geometry of our Hall bars in the ballistic transport regime, comparable to the effects observed in the so-called quenching of the Hall effect. ${ }^{14,15}$

From the above observations we know that the astonishing behavior of the huge magnetoresistance is neither caused by weak localization nor the interaction between different 2D sub-bands. The observed effect has to be related to the high mobility of the 2DEG, the corresponding mean-free path and interaction effects. 


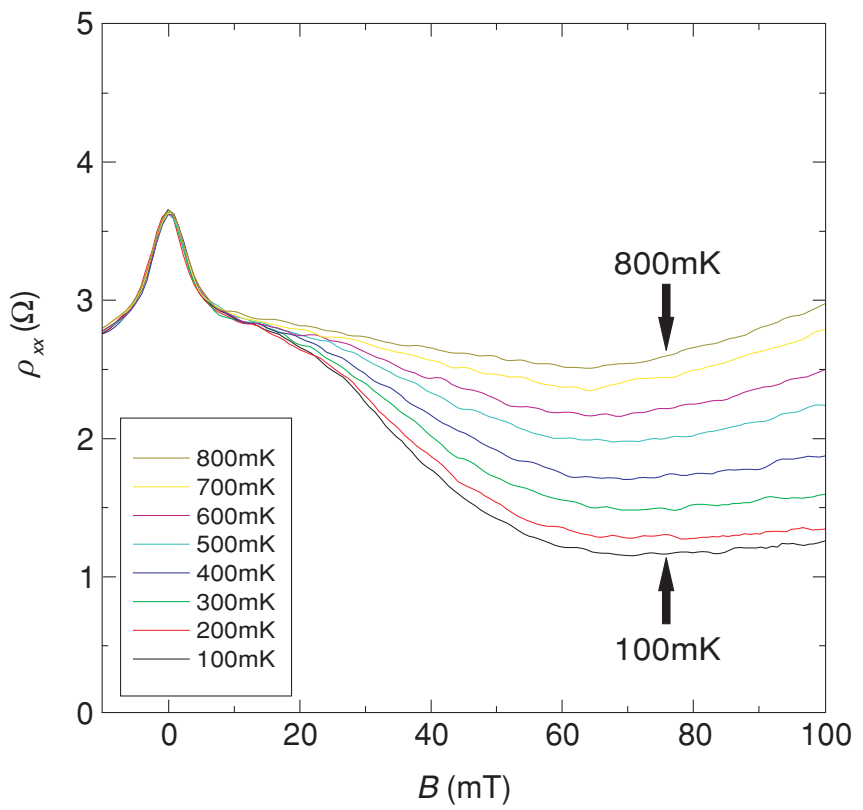

FIG. 3. (Color online) The longitudinal resistivity $\rho_{x x}$ vs. magnetic field $B$ for several temperatures, ranging between $100 \mathrm{mK}$ and $800 \mathrm{mK}$. The peak at zero magnetic field has nearly the same value for all temperatures.

To compare our measurements with others in the literaturementioned effects we examine the electron interaction correction to the conductivity $\delta \sigma_{x x}^{e e}(T) .{ }^{16-18}$ The negative magnetoresistance is in accordance with Li et al. ${ }^{18}$ expressed by

$$
\rho_{x x}=\frac{1}{\sigma_{0}}+\frac{1}{\sigma_{0}^{2}}\left(\mu^{2} B^{2}\right)\left[\delta \sigma_{x x}^{e e}(T)\right]^{-1},
$$

where $\sigma_{0}$ is the Drude conductivity. This expression includes temperature dependence and a parabolic magnetoresistance produced by long-range potential scattering. Since our sample shows $\tau>>\tau_{q}$, where $\tau_{q}$ is the quantum time determined from the magnitude of SdH oscillations to the order of magnitude of $10^{-13} \mathrm{~s}$, we can conclude that the scattering is dominated by long-range scatterers. The huge parabolic magnetoresistance is analyzed in the range of strong fields $\omega_{c} \tau>1$ which satisfy the theoretical approximations. On the basis of the transport scattering time of our samples and the equation

$$
T \geqslant \frac{\hbar}{k_{B}} \frac{1}{\tau}
$$

our measurements take place in the ballistic regime. ${ }^{17,19}$ The electron interaction induced correction to the conductivity considering the influence of the ballistic transport is then expressed by

$$
\left[\delta \sigma_{x x}^{e e}(T)\right]^{-1}=-\frac{e^{2}}{\pi h} c_{0} \alpha \sqrt{\frac{\hbar}{T \tau k_{B}}} .
$$

$c_{0}$ is according to the constant of Refs. 16-18, and has a value of about $c_{0}=0.276$. We introduce an additional factor $\alpha$ to fit our resistivities. In Fig. 2 we fit the resistivity as a $B^{2}$ dependence up to $20 \mathrm{mT}$ for different top-gate voltages and obtain $\left[\delta \sigma_{x x}^{e e}(T)\right]^{-1}$ using Eq. (1). The electron interaction

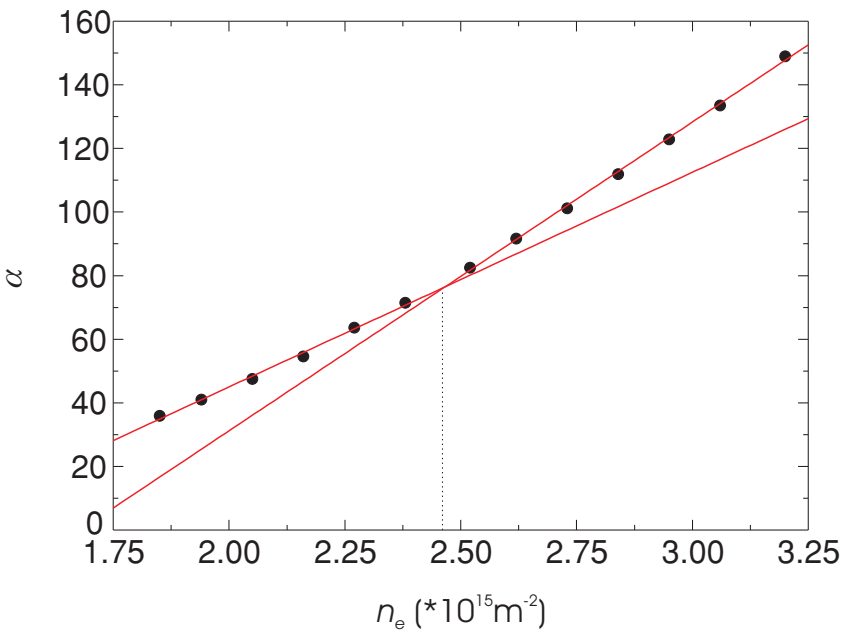

FIG. 4. (Color online) The factor $\alpha$ vs. electron density $n_{e}$. The dashed line marks the crossover from one occupied $2 \mathrm{D}$ sub-band to two occupied 2D sub-bands.

induced correction to the conductivity only fits for our measurements if $\alpha>1$. In Fig. 2 one sees that the maximum of the achieved negative parabola increases by increasing the electron density, while the curvature of the parabola is unchanged. From this observation we achieve $\alpha$ which depends strongly on the electron density and varies between 30 and 150.

Figure 4 shows the dependence of the factor $\alpha$ on the electron density. With increasing electron density $\alpha$ increases also. Between $2.4 \times 10^{11} \mathrm{~cm}^{-2}$ and $2.5 \times 10^{11} \mathrm{~cm}^{-2}$ a kink is clearly observed in the increase of the factor $\alpha$ with the density. A linear approximation for the $\alpha$ values on each side of the kink results in a crossover at $n_{e}=2.46 \times 10^{11} \mathrm{~cm}^{-2}$. Above we have shown that a second 2D sub-band is occupied for $n_{e}>2.5 \times 10^{11} \mathrm{~cm}^{-2}$. The kink marks the crossover from one occupied 2D sub-band to two occupied 2D sub-bands. Thus, the factor $\alpha$ clearly depends not only on the total electron density but also on the scattering properties of the sample which are changed for the occupation of a second 2D sub-band. ${ }^{20}$

Although we can fit the huge magnetoresistance to a parabolic magnetic field dependence as predicted for the electron interaction correction to the conductivity according to Refs. 16-18, we had to introduce this factor $\alpha$ to describe the huge magnetoresistance. From the electron interaction induced correction Eq. (3) the parabola curvature is expected to depend on $T^{-1 / 2}$. For a given gate voltage we observe that the parabola curvatures change by increasing the temperature, while the parabola maximum is unchanged (see Fig. 3). We observe a hint toward the expected temperature dependence of $T^{-1 / 2}$ for the lowest temperatures of our experimental data. Above $200 \mathrm{mK}$ the temperature dependence is more complex. A possible origin for the discrepancy between theory and experiment could be that the influence of the density fluctuation for high mobility $2 \mathrm{DEG}$ is not correctly described. In these high mobility samples the very small, but finite density variation across the sample induces an additional long range potential, up to now not treated in theory. A more sophisticated 
theoretical model of the electron interaction correction to the conductivity seems to be needed to describe these high mobility samples.

In conclusion, we observed for different gated and ungated samples a huge magnetoresistance which depends strongly on the electron density and the temperature. The huge parabolic magnetoresistance is fitted by the interaction correction to the conductivity in the situation of a long-range fluctuation potential and in the regime of ballistic transport and a discrepancy to theory is observed.

We would like to thank F. Hohls for useful discussions and for help with the experiments. This work was supported by QUEST.
*Present address: Laboratorium für Festkörperphysik, ETH Zürich, Schafmattstr. 16, 8093 Zürich, Switzerland.

${ }^{1}$ D. C. Tsui, H. L. Stormer, and A. C. Gossard, Phys. Rev. Lett. 48, 1559 (1982).

${ }^{2}$ R. B. Laughlin, Phys. Rev. Lett. 50, 1395 (1983).

${ }^{3}$ M. A. Zudov, R. R. Du, J. A. Simmons, and J. L. Reno, Phys. Rev. B 64, 201311 (2001).

${ }^{4}$ R. G. Mani, J. H. Smet, K. von Klitzing, V. Narayanamurti, W. B. Johnson, and V. Umanskyk, Nature (London) 420, 646 (2002).

${ }^{5}$ M. A. Zudov, R. R. Du, L. N. Pfeiffer, and K. W. West, Phys. Rev. Lett. 90, 046807 (2003).

${ }^{6}$ M. A. Zudov, I. V. Ponomarev, A. L. Efros, R. R. Du, J. A. Simmons, and J. L. Reno, Phys. Rev. Lett. 86, 3614 (2001).

${ }^{7}$ O. E. Raichev, Phys. Rev. B 80, 075318 (2009).

${ }^{8}$ C. L. Yang, J. Zhang, R. R. Du, J. A. Simmons, and J. L. Reno, Phys. Rev. Lett. 89, 076801 (2002).

${ }^{9}$ W. Zhang, M. A. Zudov, L. N. Pfeiffer, and K. W. West, Phys. Rev. Lett. 100, 036805 (2008).

${ }^{10}$ J. P. Eisenstein, Solid State Commun. 117, 123 (2001).
${ }^{11}$ J. P. Eisenstein, K. B. Cooper, L. N. Pfeiffer, and K. W. West, Phys. Rev. Lett. 88, 076801 (2002).

${ }^{12}$ A. R. Hamilton, E. H. Linfield, M. J. Kelly, D. A. Ritchie, G. A. C. Jones, and M. Pepper, Phys. Rev. B 51, 17600 (1995).

${ }^{13}$ S. Syed, M. J. Manfra, Y. J. Wang, R. J. Molnar, and H. L. Stormer, Appl. Phys. Lett. 84, 1507 (2004).

${ }^{14}$ M. L. Roukes, A. Scherer, S. J. Allen Jr., H. G. Craighead, R. M. Ruthen, E. D. Beebe, and J. P. Harbison, Phys. Rev. Lett. 59, 3011 (1987).

${ }^{15}$ T. J. Thornton, M. L. Roukes, A. Scherer, and B. P. VandeGaag, Phys. Rev. Lett. 63, 2128 (1989).

${ }^{16}$ M. A. Paalanen, D. C. Tsui, and J. C. M. Hwang, Phys. Rev. Lett. 51, 2226 (1983).

${ }^{17}$ I. V. Gornyi and A. D. Mirlin, Phys. Rev. Lett. 90, 076801 (2003).

${ }^{18}$ L. Li, Y. Y. Proskuryakov, A. K. Savchenko, E. H. Linfield, and D. A. Ritchie, Phys. Rev. Lett. 90, 076802 (2003).

${ }^{19}$ I. V. Gornyi and A. D. Mirlin, Phys. Rev. B 69, 045313 (2004).

${ }^{20}$ H. L. Stormer, A. C. Gossard, and W. Wiegmann, Solid State Commun. 41, 707 (1982). 